\title{
A Multicenter Pilot Study on the Clinical Utility of Computational Modeling for Flow-Diverter Treatment Planning
}

(D)B.W. Chong, (D)B.R. Bendok, C. Krishna, (D) M. Sattur, (D)B.L. Brown, (DR.G. Tawk, DD.A. Miller, (D) Rangel-Castilla, (DH. Babiker,

(D) D.H. Frakes, (D) A. Theiler, (D) H. Cloft, DD. Kallmes, and (D) G. Lanzino

\begin{abstract}
BACKGROUND AND PURPOSE: Selection of the correct flow-diverter size is critical for cerebral aneurysm treatment success, but it remains challenging due to the interplay of device size, anatomy, and deployment. Current convention does not address these challenges well. The goals of this pilot study were to determine whether computational modeling improves flow-diverter sizing over current convention and to validate simulated deployments.
\end{abstract}

MATERIALS AND METHODS: Seven experienced neurosurgeons and interventional neuroradiologists used computational modeling to prospectively plan 19 clinical interventions. In each patient case, physicians simulated 2-4 flow-diverter sizes that were under consideration based on preprocedural imaging. In addition, physicians identified a preferred device size using the current convention. A questionnaire on the impact of computational modeling on the procedure was completed immediately after treatment. Rotational angiography image data were acquired after treatment and compared with flow-diverter simulations to validate the output of the software platform.

RESULTS: According to questionnaire responses, physicians found the simulations useful for treatment planning, and they increased their confidence in device selection in $94.7 \%$ of cases. After viewing the simulations results, physicians selected a device size that was different from the original conventionally planned device size in $63.2 \%$ of cases. The average absolute difference between clinical and simulated flow-diverter lengths was $2.1 \mathrm{~mm}$. In $57 \%$ of cases, average simulated flow-diverter diameters were within the measurement uncertainty of clinical flow-diverter diameters.

CONCLUSIONS: Physicians found computational modeling to be an impactful and useful tool for flow-diverter treatment planning. Validation results showed good agreement between simulated and clinical flow-diverter diameters and lengths.

ABBREVIATION: $F D$ = flow diverter

$\mathbf{F}$ ow diverters (FDs) are being used with increasing frequency for the treatment of cerebral aneurysms. The immediate goal of FD treatment is to promote hemodynamic stasis and thrombus formation within the aneurysmal sac via flow diversion. Several studies have shown impressively effective use of FD devices in treating small-to-large aneurysms. ${ }^{1-3}$ Recently, the FDA also approved the expanded indication for FD products for a much wider range of aneurysm sizes and locations, paving the way for

Received April 1, 2019; accepted after revision August 5.

From the Department of Neurosurgery (B.W.C., B.R.B., C.K., M.S.), Mayo Clinic, Phoenix, Arizona; Department of Neurosurgery (B.L.B., R.G.T., D.A.M.), Mayo Clinic, Jacksonville, Florida; Department of Neurosurgery (L.R.-C., A.T., H.C., D.K., G.L.), Mayo Clinic, Rochester, Minnesota; Endovantage, LLC (H.B.), Phoenix, Arizona; and Department of Biological and Health Systems Engineering (B.W.C., D.H.F.), Arizona State University, Tempe, Arizona.

This study was funded by the Mayo Clinic Office of Translation to Practice.

Paper previously presented, in part, at: Western Neuroradiological Society Annual Meeting, October 11-14, 2018, Dana Point, California; and SNIS 15TH Annual

Meeting, July 23-26, 2018, San Francisco, California. additional FD entries into the market. ${ }^{4}$ Nevertheless, selection of the correct FD size remains challenging and is an important consideration in the context of treatment success. Oversizing FDs can lead to in-stent stenosis or poor device expansion, ${ }^{5,6}$ while undersizing can lead to device migration, prolapse into the aneurysm, and/or poor vessel coverage. ${ }^{7,8}$ Deploying FDs in highly curved vessels can also present a number of technical challenges. ${ }^{9}$

The conventional approach to sizing FDs begins with measuring vessel diameters in images at the desired proximal and distal landing points. Specifically, lines projected onto $2 \mathrm{D}$ images are used to quantify the diameters. Next, the vessel length between

Please address correspondence to Brian W. Chong, MD, Mayo Clinic Hospital, Clinical Studies Unit, 5777 East Mayo Blvd, Phoenix, AZ 85017; e-mail: Chong.Brian@mayo.edu

$\checkmark$ Indicates article with supplemental on-line video.

三 Indicates article with supplemental on-line tables.

http://dx.doi.org/10.3174/ajnr.A6222

AJNR Am J Neuroradiol 40:1759-65 Oct 2019 www.ajnr.org 
the 2 points is estimated. These collective measurements are used to select an FD size that will hopefully appose well to the vessel wall and cover the deployment region. However, this approach to sizing can be challenging because vessel diameters may vary considerably along the trajectory of a vessel. FDs can also elongate by $>50 \%$ of the nominal length indicated by labeling. ${ }^{10}$ Furthermore, measurements of vessel size taken from angiographic image data can be operator-dependent and are prone to measurement error. A recent study on intra- and interobserver variability when measuring cerebral aneurysm size in CT and MR angiography images showed a standard difference range of $12 \%-18 \%$ in size measurements. ${ }^{11}$ A similar range in measurement variability during pretreatment planning can potentially lead to substantial FD undersizing or oversizing.

At present, there is no established method for predicting FD behavior in a patient's anatomy.

The physician's experience plays a primary role in understanding the interplay between device size, anatomy, and deployment. Different techniques for repairing suboptimal deployments have also been reported in the literature, including the use of catheter maneuvers, balloons, and additional FDs. ${ }^{8,12,13}$ Needless to say, avoiding complications and suboptimal FD deployments through better planning and sizing is preferable to compensating for poor outcomes.

Computational modeling can help predict FD deployment behavior and thereby improve planning and selection of the optimal predicted FD size for a given patient. The use of computational modeling in clinical practice has grown widely during the past few years. ${ }^{14,15}$ Here we present a pilot study that investigates the use of a computational modeling platform for the planning of FD treatments. The goals of the study were to determine whether computational modeling improves the selection of FD sizes over current convention and to validate the simulated deployments. The study surveys 7 experienced neurosurgeons and neurointerventional radiologists who used the proposed software platform to prospectively plan clinical interventions, and it validates the output of the process against rotational angiography scans of the actual clinical deployments.

\section{MATERIALS AND METHODS \\ Patient Demographics}

Mayo Clinic review board approval was obtained for the study protocol and patient recruitment. Eligible patients who provided written informed consent and met the inclusion criteria were included in the study. The study was conducted according to the Health Insurance Portability and Accountability Act. The primary inclusion criterion was patients approved for treatment with the Pipeline Embolization Device (PED; Covidien, Irvine, California). The major exclusion criteria were contraindications to $3 \mathrm{D}$ rotational angiography, insufficient time for treatment planning (at least 48 hours were required in the study), and poor image quality for vessel reconstruction (eg, poor contrast in the vessel or major artifacts near the aneurysm including prior coiling). Forty-three unruptured intracranial aneurysms that were approved for treatment with the Pipeline device at 3 Mayo Clinic institutions between January 2017 and July 2019 were included as eligible cases in the study. However, 16 cases were withdrawn due
Table 1: Number of cases simulated and validated by physicians

\begin{tabular}{lcc}
\hline Physician & Institution & $\begin{array}{c}\text { No. of Cases } \\
\text { Simulated/Validated }\end{array}$ \\
\hline 1 & $\mathrm{~A}$ & $1 / 1$ \\
2 & $\mathrm{~A}$ & $7 / 6$ \\
3 & $\mathrm{~A}$ & $6 / 5$ \\
4 & $\mathrm{~B}$ & $1 / 1$ \\
5 & $\mathrm{~B}$ & $1 / 0$ \\
6 & $\mathrm{~B}$ & $1 / 0$ \\
7 & $\mathrm{C}$ & $2 / 1$ \\
\hline
\end{tabular}

to clinical or administrative reasons (eg, the Pipeline device was not used, or forms were not received), 4 were withdrawn because of artifacts in the image data or poor image quality, and 4 were withdrawn due to an insufficient time for the computational modeling process (eg, when patient care needs precluded a 48hour simulation time). The remaining 19 cases were included in the study. Each patient case represented a single aneurysm that was treated.

In total, 7 neurosurgeons and interventional neuroradiologists participated in the study. Five of the physicians had $>15$ years of experience in endovascular cerebral aneurysm treatments, while 2 had between 5 and 7 years of experience. Table 1 presents the number of cases performed by each physician. Patient demographics, clinical presentation, aneurysm location and size, pretreatment image data, and procedural information were acquired for each patient case.

\section{Treatment-Planning Workflow}

The SurgicalPreview computational modeling software (Endovantage, Phoenix, Arizona) was used for FD planning. The software is cleared by the FDA for computational modeling of the placement and deployment of neurointerventional devices. ${ }^{16}$ Rotational angiography image data were acquired for each case before treatment and uploaded to the SurgicalPreview Web portal for vessel reconstruction and translation into a computational model. The reconstruction process was performed by trained Endovantage personnel using a thresholding-based, semiautomated image-segmentation technique. The $3 \mathrm{D}$ reconstructed models were uploaded back to the SurgicalPreview portal after reconstruction. The physician treating the case then indicated a distal landing point on the $3 \mathrm{D}$ vessel model in the portal for $\mathrm{FD}$ simulations and selected 2-4 Pipeline sizes for consideration from a list of all commercially available Pipeline sizes. Physicians used conventional vessel measurements on the patient's image data to identify potential FD sizes. They were also required to identify a preferred device size before viewing the simulation results. Selecting a single device in this way aligns with the current convention and facilitates later evaluations of the impact of computational modeling on final device selection.

FD simulations were performed automatically in SurgicalPreview on a high-performance computing cluster. The simulations use the finite element method and clinical deployment strategy to simulate deployment. ${ }^{17}$ In summary, the software models a virtual catheter with the same diameter as the Pipeline delivery system and a linear-elastic material model to characterize the mechanical characteristics of the microcatheter. The geometry of all Pipeline FD sizes is modeled according to manufacturer's 
specifications, and linear-elastic material models are used to characterize the material properties of the cobalt-chromium and platinum-tungsten alloy wires in the devices. Physical radialcompression mechanical tests were performed by Endovantage for each Pipeline FD size to calibrate the linear-elastic material models. The vessel model is assumed to be rigid, and the deployment procedure is assumed to be quasi-static. The software virtually deploys the FD into a vessel using an explicit finite element simulation. A feedback loop is used during the simulation to automatically adjust the deployment according to the vessel diameter and the diameter of the unsheathed region of the FD. This virtual feedback loop models the clinical deployment strategy of "painting the vessel wall" with the FD and promotes favorable apposition during deployment.

Results of simulating different FD sizes were then disseminated to each physician for feedback before treatment. The results included 3D models of the simulated FDs inside the pretreatment vessel, a deployment video showing unsheathing of the FD, and quantitative plots showing cross-sections of the vessel and FD at points that are uniformly spaced by $0.2 \mathrm{~mm}$ along the vessel centerline. A sample simulation result for $1 \mathrm{FD}$ size is provided in Fig 1. An On-line Video of a simulated deployment showing unsheathing of the FD is also provided. Evaluations of the simulation results were performed by physicians at their offices, in their reading rooms, or before treatment in the operating room.

Each physician completed a questionnaire immediately after treatment that detailed procedural notes, devices used, and procedural time. The questionnaire also contained 5 questions on the impact of computational modeling on the procedure. Those questions are provided in Table 2. A clinical coordinator ensured that all fields were filled out.
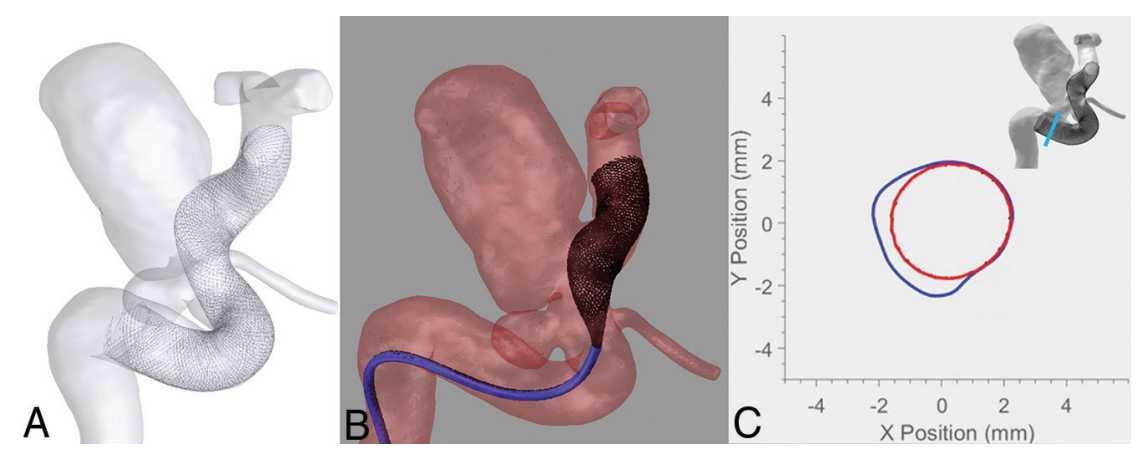

FIG 1. Sample simulation result from the SurgicalPreview computational modeling software showing a 3D model of the FD inside a pretreatment vessel $(A)$, a frame from a deployment video $(B)$, and a cross-section of the FD (red) and vessel (blue) at a position along the vessel centerline (C).

\section{Deployment Validations}

To validate the simulation results, we acquired a rotational angiography scan after each procedure. In total, posttreatment data were acquired for 15 of the 19 cases that were prospectively planned. The image data were used to reconstruct the clinically deployed FD devices and compare them with simulated deployments. Mimics (Materialise, Leuven, Belgium) was used to perform the reconstruction. An additional FD simulation was performed in the pretreatment vessel using the device model of the actual FD size used in the procedure, deployed at the exact distal landing point observed in the posttreatment reconstructions. The additional simulations addressed cases in which the physician used feedback from the simulation results to select a different device size and/or cases characterized by large discrepancies between the physician's desired and actual distal landing points.

A centerline that was discretized with $0.1-\mathrm{mm}$ resolution was generated for each simulated and reconstructed FD using the Vascular Modeling Toolkit (VMTK, www.vmtk.org). At each point along the centerline, VMTK also computed the maximal inscribed sphere that could be fit at that location. FD length was calculated by measuring the total length of each simulated and clinical FD centerline. The average FD diameter was calculated by averaging the diameters of all maximal inscribed spheres along the length of each centerline.

The calculation of the average FD diameter was made using the outer surfaces of the reconstructed clinical and simulated FDs (ie, the inner lumen was ignored). In the reconstructed deployments, the outer FD surface is affected by the spatial image resolution and blooming artifact that is introduced by the actual FD in the posttreatment image data. The artifact expands device thickness beyond the actual thickness of the device and adds uncertainty to the measurement of FD diameter. ${ }^{18,19}$ To quantify that uncertainty, we made 15 measurements of FD wire thickness (inner lumen to outer surface) at random locations along the entire length of each clinical FD using a digital caliper in Geomagic Studio (3D Systems, Rock Hill, South Carolina). The 15 measurements were averaged in each deployment and used to define the uncertainty range in measuring the clinical FD diameter. The true clinical FD diameter was assumed to be near the center of that uncertainty range.

Table 2: Survey questionnaire results on the impact of computational modeling

\begin{tabular}{|c|c|c|c|}
\hline \multirow[b]{2}{*}{ Survey Questions } & \multicolumn{3}{|c|}{ Responses } \\
\hline & Yes & No & Somewhat \\
\hline Were the simulations useful for your planning? & 18 & 0 & 1 \\
\hline Did the simulations give you greater confidence in your device selection? & 18 & 0 & 1 \\
\hline Did the simulations change your device selection? & 12 & 6 & 1 \\
\hline Do you think the simulations reduced the number of devices that you used? & 2 & 14 & 3 \\
\hline Do you think the simulations reduced your operative time? & 4 & 13 & 2 \\
\hline
\end{tabular}


After the calculation of clinical and simulated FD lengths and diameters, a statistical analysis was performed to evaluate the differences between the deployments. Specifically, the mean, SD, and $95 \%$ confidence interval of the average difference and average absolute difference between deployments were calculated.

\section{RESULTS}

\section{Patient Demographics}

Of the 19 cases that were simulated in the study, 4 had presented with a different cerebral aneurysm previously. Nine patients were current or previous smokers. Most aneurysms were located on the internal carotid artery, and only 2 of the 19 aneurysms were fusiform, while the remaining were saccular. Regarding the sizes of the 17 saccular aneurysms, 6 were $<7 \mathrm{~mm}$, 9 were $7-10 \mathrm{~mm}$, and 2 were $>10 \mathrm{~mm}$, while the measurement in millimeters refers to the largest dimension of the aneurysm. The average saccular aneurysm size was $8.4 \pm 4.0 \mathrm{~mm}$. The largest dimensions of the 2 fusiform aneurysms were 8.3 and $7.0 \mathrm{~mm}$.

\section{Physician Responses}

Table 2 presents the results of the surgical-planning questionnaire. Physicians found the simulations useful for treatment planning in $94.7 \%$ of cases (ie, 18/19). Their comments on simulation utility in the questionnaire said that the simulations were specifically useful for the following: 1) rehearsing the deployment strategy and positioning of the device, 2) predicting how the device would behave around curved regions in the vessel, and 3) narrowing the list of device sizes being considered. Responses also indicated that the simulations increased perceived confidence in device selection in $94.7 \%$ of cases (ie, 18/19). Some physicians commented that they became more confident that the selected device size would span the desired proximal and distal landing points after viewing the simulation results.

After viewing the simulation results, physicians selected a device size that was different from the originally planned device size in $63.2 \%$ of cases (ie, 12/19). In most of those cases (9/12), physicians selected a device diameter or length that was 1 size smaller or larger than the originally planned device size. Furthermore, a different device diameter was selected in half of the cases $(6 / 12)$, while only a different device length was selected in the remaining half. According to some questionnaire comments, physicians chose a different device length after viewing the simulated proximal landing point or chose a different device diameter on the basis of the simulated FD coverage of the vessel wall.

Physicians perceived that the simulations reduced the number of devices used and operative time in $10.5 \%$ (ie, 2/19) and $21.1 \%$ of cases (ie, 4/18), respectively. Seventeen of the 19 cases were treated with a single Pipeline device, while only 2 cases were treated with 2 Pipeline devices (ie, telescoping configuration). Balloon angioplasty was used in 2 cases, once to expand the proximal end of the FD after it did not fully open and once to expand the device to achieve better wall apposition. Procedural complications occurred in 2 cases. In 1 case, complete occlusion of the internal carotid artery with recanalization was observed, and in the second case, cerebral vasospasm was encountered. Regarding the procedural times of the 19 cases (quantified at the time from puncture until complete deployment), 6 cases were $<30$ minutes,
9 cases were $30-60$ minutes, and 4 cases were $>60$ minutes. The average procedural time was $41.5 \pm 19.4$ minutes.

\section{Deployment Validations}

Fifteen of the 19 cases had posttreatment image data that could be used for validation analysis. However, 1 case was excluded because balloon angioplasty was used to expand the FD to achieve better wall apposition. Accordingly, 14 cases were considered in the validation analysis. Figure 2 shows 6 pairs of clinical and simulated deployments and presents them in ascending order according to their difference in device length. The figure shows similarity between predicted and actual FD deployments based on device length and diameter.

Figure 3 presents Bland-Altman plots that compare FD lengths and diameters between clinical and simulated deployments, and Table 3 presents the statistical analysis of the difference between deployments. On average, the simulations were $1.1 \mathrm{~mm}$ longer and $0.1 \mathrm{~mm}$ larger in diameter than clinical deployments. A 95\% confidence interval indicated that the true average absolute difference in FD length was between 1.3 and $2.9 \mathrm{~mm}$. The $95 \%$ confidence interval for the true average absolute difference in FD diameter was between 0.18 and $0.37 \mathrm{~mm}$, which is close to the range of image spatial resolutions for rotational angiography data ${ }^{11}$ and the range of the measured uncertainty in FD diameter measurements $(0.13-0.39 \mathrm{~mm})$. Figure 4 shows that in $57 \%$ of cases, the simulations had an average diameter that was within the uncertainty of the clinical FD diameter measurement. Note that wire thickness was used to quantify the uncertainty in clinical FD diameter measurements due to blooming artifacts and the spatial resolution of the posttreatment image data.

Additional detail on patient demographics, physician responses, and deployment validation are provided in the On-line Tables 1-4.

\section{DISCUSSION}

Technical complications such as malapposition, prolapse, migration, and incomplete expansion are common during FD deployment. Reports of such complications have increased recently due to the frequent use of FDs and improvement to the imaging technology used to visualize the devices after deployment. ${ }^{20,21}$ To date, the rate of technical complications has ranged from $12 \%$ to $18 \% .^{13,20,21}$ The recommended strategy for avoiding these complications is to select a FD size that will appose well to the vessel wall and cover at least $2-3 \mathrm{~mm}$ of the parent vessel beyond the aneurysm region. ${ }^{22}$ Good wall apposition is also crucial for vessel remodeling and aneurysmal occlusion and may be a key factor for achieving complete occlusion as well as avoiding thrombosis of perforator branches. ${ }^{23,24}$ Accordingly, proper FD sizing is critical for interventional success.

This study found computational modeling to be clinically useful for FD sizing and procedural planning. The participating physicians were able to experiment with different device sizes before interventions and select the predicted best option for a given patient's anatomy. According to questionnaire responses, physicians not only thought that modeling was useful for sizing but also found that it improved their confidence in device 


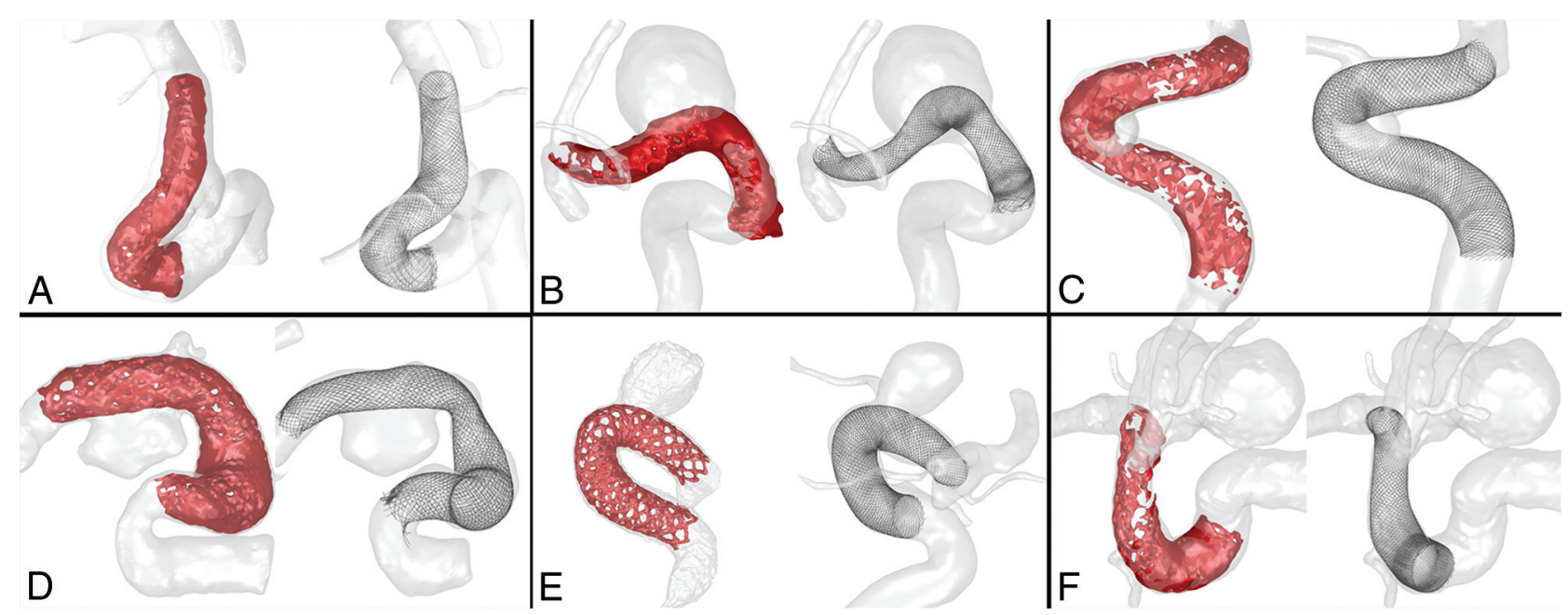

FIG 2. Examples of posttreatment clinical reconstructions (red/left) and pretreatment simulation results (black/right) for the same FD sizes. The deployment pairs are sorted in ascending order according to the difference between actual and simulated device lengths, which ranged from 1.58 to $4.06 \mathrm{~mm}$.

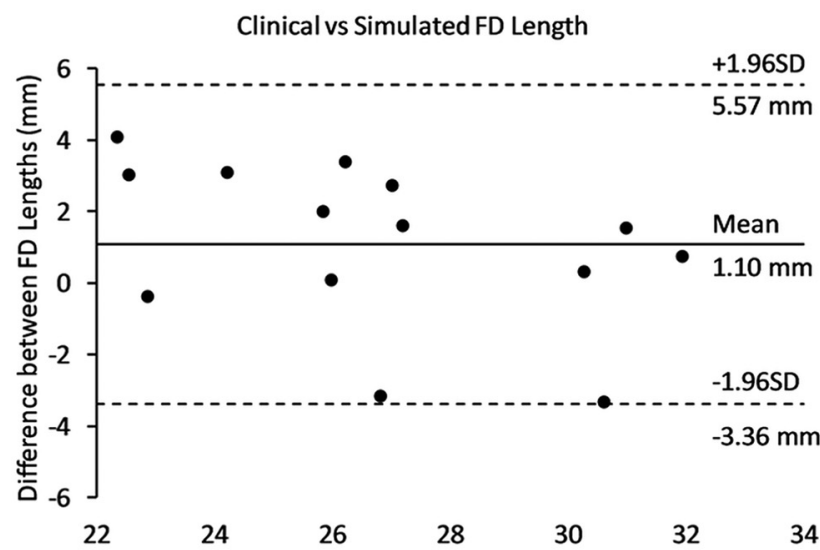

A

Mean FD length (mm)

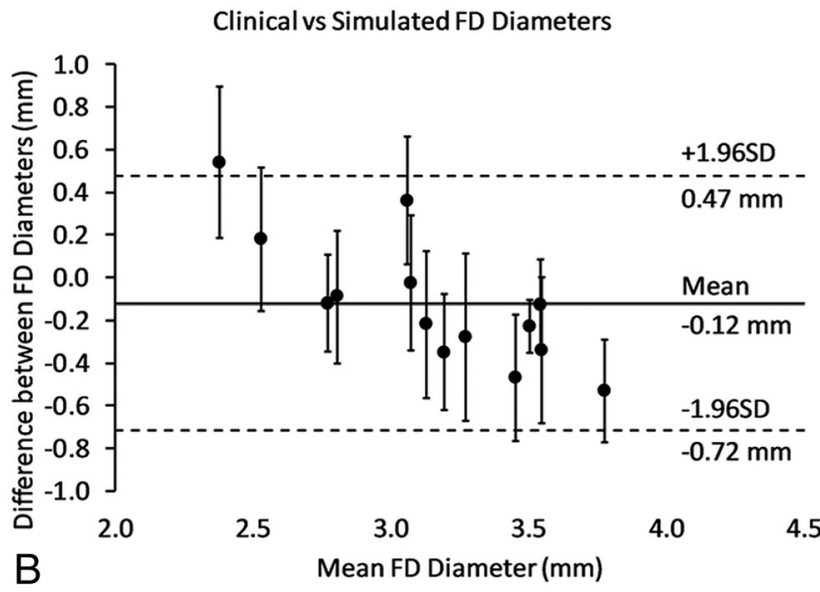

FIG 3. Bland-Altman plots showing dots that represent differences between the actual clinical and simulated deployments in FD length $(A)$ and FD diameter $(B)$. The plots show the means for the deployment pairs on the $x$-axis and the differences between pairs on the $y$-axis.

Table 3: Statistical analysis of the difference between clinical and simulated FD lengths and diameters for the 14 validated cases

\begin{tabular}{|c|c|c|c|c|c|c|}
\hline & \multicolumn{3}{|c|}{ Difference } & \multicolumn{3}{|c|}{ Absolute Difference } \\
\hline & Mean $(\mathrm{mm})$ & $\mathrm{SD}(\mathrm{mm})$ & $95 \% \mathrm{Cl}(\mathrm{mm})$ & Mean (mm) & $\mathrm{SD}(\mathrm{mm})$ & $95 \% \mathrm{Cl}(\mathrm{mm})$ \\
\hline FD length & 1.10 & 2.28 & {$[-0.21,2.42]$} & 2.09 & 1.34 & {$[1.32,2.86]$} \\
\hline FD diameter & -0.12 & 0.30 & {$[-0.30,0.05]$} & 0.28 & 0.16 & {$[0.18,0.37]$} \\
\hline
\end{tabular}

placement. This effect was observed for both small $(<7 \mathrm{~mm})$ and large $(>7 \mathrm{~mm})$ aneurysms. Deployment videos of FD simulations helped some physicians to mentally rehearse the procedure and visualize the behavior of FDs around tortuous vessels. After viewing simulation results, the participating physicians changed their device size selections in $63.2 \%$ of the cases. This result highlights the impact of computational modeling on treatment planning.

Physician responses indicated that the effects of computational modeling on the number of devices used and on procedural time were limited to a small percentage of cases. However, such effects may be difficult to observe on the basis of a questionnaire, given a small sample size. A planned future study will use a larger patient population and a control group to better elucidate the influence of computational modeling on the number of devices used and procedural time.

Validation analysis showed good agreement between simulated and actual clinical FD diameters and lengths. Most of the simulations had average FD diameters that were within the uncertainty of the clinical FD diameter measurements, and in some cases, dissimilarity between the deployment technique of the software and the physician's technique led to FD length differences of $>3 \mathrm{~mm}$. Nevertheless, an average absolute difference of only $2.1 \mathrm{~mm}$ in FD length was observed over all cases examined. 
Average FD Diameter

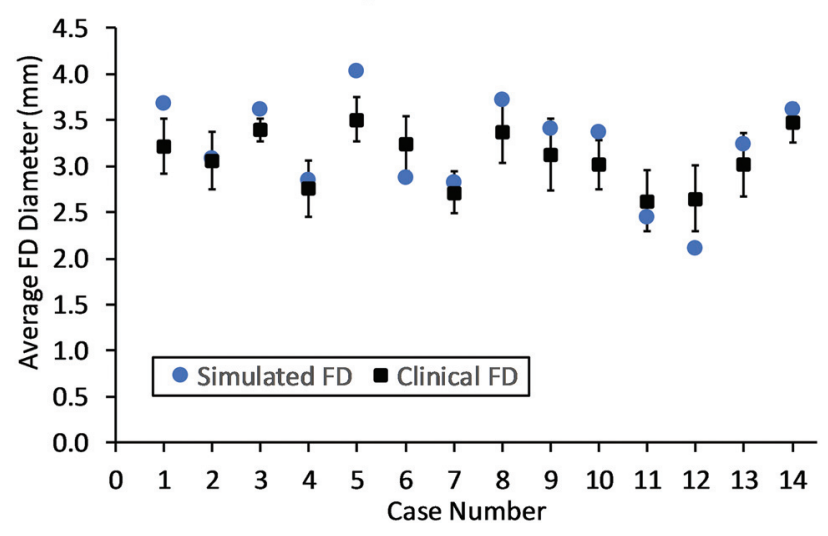

FIG 4. Average FD diameters for the actual clinical and simulated deployments. Error bars in the clinical FD diameter measurements were calculated by averaging 15 random measurements of FD thickness along the length of each reconstructed FD model.

Many studies have been reported in the literature on the application of computational device modeling to treatment planning. ${ }^{10,25-27}$ Previous retrospective studies that evaluated the application of computational modeling to FD sizing have demonstrated the potential benefits of the technology for improving FD size selection. ${ }^{10,25}$ To date, few, if any, studies have exclusively focused on both the utility and predictive fidelity of computational device modeling in a prospective case series. Yet, evaluations under typical clinical workflows are essential for understanding the contribution of the technology to clinical practice and its potential for adoption. This study presents an early evaluation of the impact of the technology on FD treatment planning.

Several limitations of this pilot study are noteworthy. First, the study included a small sample of patient cases and a small number of physicians. The small sample size was a reflection of the fact that several cases were referred from outside hospitals and these patients already had a recent CTA or catheter angiograms of their aneurysms. We did not think that it was justified to repeat the imaging and thereby expose the patients to additional unnecessary radiation. Therefore, these patients were excluded from enrollment. Furthermore, the primary goal of the study was to gauge the impact of computational modeling on clinical workflows, and a sample size of 19 was deemed appropriate for this purpose. Future work will include evaluating the impact of computational modeling on a larger patient population with a control group. Second, the distribution of cases among physicians was skewed, and most cases $(73.6 \%)$ were performed by physicians operating at one of three sites (all part of the same healthcare organization). This distribution is proportional to the size of the site and the volume of Pipeline cases that the site receives. Therefore most cases were performed by the physicians from the largest and highest volume site. Third, the study did not evaluate short- and long-term patient outcomes such as aneurysmal occlusion, which is another goal of future work. Fourth, the study used a self-assessment questionnaire to evaluate the perceived usefulness of the technology. Self-assessment alone may not be an accurate measure of the usefulness or the improvement of the technology over convention and should be supported by performance metrics from a larger study with a control group.

\section{CONCLUSIONS}

Computational modeling of FDs was found to be an impactful clinical tool for interventional planning. Experienced interventional neuroradiologists and neurosurgeons at 3 centers found the modeling to be useful for FD sizing, FD placement, and case rehearsal. Use of the modeling platform led to device selection changes in $63.2 \%$ of cases. Validation analysis showed good agreement between simulated and actual clinical FD diameters and lengths. The proposed computational modeling approach has the potential to reduce technical complications during FD treatment and improve patient outcomes. To our knowledge, this is the first study that evaluates the utility of computational device modeling in a prospective case series.

Disclosures: Brian W. Chong—RELATED: Board Membership: Endovantage LLC; Patents (Planned, Pending, Or Issued): Mayo Clinic, Comments: patent pending on intellectual property related to work on the article; Royalties: Mayo Clinic, Comments: royalty sharing with Mayo Clinic; Stock/Stock Options: Endovantage LLC; Support for Travel to Meetings for the Study or Other Purposes: Endovantage LLC, Comments: travel (airfare and hotel) to present at a scientific meeting. Haithem Babiker-RELATED: Employment: Endovantage LLC; Patents (Planned, Pending or Issued): Endovantage LLC; Stock/Stock Options: Endovantage LLC. David Kallmes-UNRELATED: Consultancy: Medtronic, Comments: Safety Board for flow-diverter clinical trial*; Grants/Grants Pending: Medtronic, Sequent, MicroVention, NeuroSigma, Comments: support for preclinical and clinical research*; Stock/Stock Options: Superior Medical Experts, Comments: founder and stockholder of a medical editing and writing business. Giuseppe LanzinoUNRELATED: Consultancy: Superior Medical Editing. Rabih G. TawkUNRELATED: Stock/Stock Options: Medtronic, Comments: \$5000-\$10,000. *Money paid to institution.

\section{REFERENCES}

1. Briganti F, Delehaye L, Leone G, et al. Flow diverter device for the treatment of small middle cerebral artery aneurysms. $J$ NeuroIntervent Surg 2016;8:287-94 CrossRef Medline

2. Bhogal P, Pérez MA, Ganslandt O, et al. Treatment of posterior circulation non-saccular aneurysms with flow diverters: a single-center experience and review of $\mathbf{5 6}$ patients. J NeuroIntervent Surg 2017;9:471-81 CrossRef Medline

3. Saatci I, Yavuz K, Ozer C, et al. Treatment of intracranial aneurysms using the Pipeline flow-diverter embolization device: a single-center experience with long-term follow-up results. AJNR Am J Neuroradiol 2012;33:1436-46 CrossRef Medline

4. U.S. Food and Drug Administration. P100018/S015, Pipeline ${ }^{\mathrm{TM}}$, Flex Embolization Device approval letter. https://www.accessdata. fda.gov/cdrh_docs/pdf10/P100018S015A.pdf. Accessed May 22, 2019

5. Caroff J, Tamura T, King RM, et al. Phosphorylcholine surface modified flow diverter associated with reduced intimal hyperplasia. J NeuroIntervent Surg 2018;10:1097-1101 CrossRef Medline

6. Laurent E, Makoyeva A, Darsaut TE, et al. In vitro reproduction of device deformation leading to thrombotic complications and failure of flow diversion. Interv Neuroradiol 2013;19:432-37 CrossRef

7. Tsai YH, Wong HF, Hsu SW. Endovascular management of spontaneous delayed migration of the flow-diverter stent. J Neuroradiol 2018 Dec 1. [Epub ahead of print] CrossRef Medline

8. Al-Mufti F, Amuluru K, Cohen ER, et al. Rescue therapy for procedural complications associated with deployment of flow-diverting devices in cerebral aneurysms. Oper Neurosurg (Hagerstown) 2018; 15:624-33 CrossRef Medline 
9. Sindeev S, Prothmann S, Frolov S, et al. Intimal hyperplasia after aneurysm treatment by diversion. World Neurosurg 2019;122:e577-83 CrossRef Medline

10. Narata AP, Blasco J, Roman LS, et al. Early results in flow diverter sizing by computational simulation: quantification of size change and simulation error assessment. Oper Neurosurg (Hagerstown) 2018;15:557-66 CrossRef Medline

11. Kim HJ, Yoon DY, Kim ES, et al. Intraobserver and interobserver variability in CT angiography and MR angiography measurements of the size of cerebral aneurysms. Neuroradiology 2017;59:491-97 CrossRef Medline

12. Martínez-Galdámez M, Ortega-Quintanilla J, Hermosín A, et al. Novel balloon application for rescue and realignment of a proximal end migrated Pipeline Flex embolization device into the aneurysmal sac: complication management. BMJ Case Rep 2016;2016 CrossRef Medline

13. Kühn AL, Rodrigues KDM, Wakhloo AK, et al. Endovascular techniques for achievement of better flow diverter wall apposition. Interv Neuroradiol 2019;25:344-47 CrossRef Medline

14. Pontone G, Patel MR, Hlatky MA, et al. Rationale and design of the prospective longitudinal trial of FFRCT: outcome and resource impacts study. Am Heart J 2015;170:438-44 CrossRef Medline

15. Brouwer J, Gheorghe L, Nijenhuis VJ, et al. Insight on patient specific computer modeling of transcatheter aortic valve implantation in patients with bicuspid aortic valve disease. Catheter Cardiovasc Interv 2019;93:1097-1105 CrossRef Medline

16. U.S. Food and Drug Administration. K171534, Surgical Preview. clearance letter. https://www.accessdata.fda.gov/cdrh_docs/pdf17/ K171534.pdf. Accessed May 16, 2019

17. Babiker H, Kalani $Y$, Levitt $M$, et al. Clinical validations of simulated neurovascular braided stent deployments. Ann Biomed Eng 2016;44:3719-49 CrossRef

18. Zwarzany $€$, Poncyljusz W, Burke TH. Flat detector CT and its applications in the endovascular treatment of wide-necked intracranial aneurysms: a literature review. Eur J Radiol 2017; 88:26-31 CrossRef Medline
19. Ott S, Struffert T, Saake M, et al. Influence of different reconstruction parameters in the visualization of intracranial stents using C-arm flat panel CT angiography: experience in an animal model. Acta Radiology 2016;57:233-40 CrossRef Medline

20. Kizilkilic O, Kocer N, Metaxas GE, et al. Utility of VasoCT in the treatment of intracranial aneurysm with flow-diverter stents: clinical article. J Neurosurg 2012;117:45-49 CrossRef Medline

21. Clarençon F, Di Maria F, Gabrieli J, et al. Clinical impact of flat panel volume CT angiography in evaluating the accurate intraoperative deployment of flow-diverter stents. AJNR Am J Neuroradiol 2017;38:1966-72 CrossRef Medline

22. Al-Mufti F, Cohen ER, Amuluru K, et al. Bailout strategies and complications associated with the use of flow-diverting stents for treating intracranial aneurysms. Interv Neuroradiol 2019;8:38-54 CrossRef

23. Rouchaud A, Ramana C, Brinjikji W, et al. Wall apposition is a key factor for aneurysm occlusion after flow diversion: a histologic evaluation in 41 rabbits. AJNR Am J Neuroradiol 2016;37:2087-91 CrossRef Medline

24. Aquarius R, de Korte A, Smits D, et al. The importance of wall apposition in flow diverters. Neurosurgery 2019;84:804-10 CrossRef Medline

25. Ospel JM, Gascou G, Costalat V, et al. Comparison of Pipeline embolization device sizing based on conventional $2 \mathrm{D}$ measurements and virtual simulation using the Sim\&Size software: an agreement study. AJNR Am J Neuroradiol 2019;40:524-30 CrossRef Medline

26. Karády J, Ntalas I, Prendergast B, et al. Transcatheter mitral valve replacement in mitral annulus calcification: the art of computer simulation. J Cardiovasc Comput Tomogr 2018;12:153-57 CrossRef Medline

27. Derycke L, Perrin D, Cochennec F, et al. Predictive numerical simulations of double branch stent-graft deployment in an aortic arch aneurysm. Ann Biomed Eng 2019;47:1051-62 CrossRef Medline 